\title{
The correlations between the anchor density and the curve correction of adolescent idiopathic scoliosis surgery
}

\author{
Yu-Cheng Yeh ${ }^{1,2,3}$, Chi-Chien Niu¹,2,3, Lih-Huei Chen ${ }^{1,2,3}$, Wen-Jer Chen ${ }^{4}$ and Po-Liang Lai ${ }^{1,2,3^{*}}$ (D)
}

\begin{abstract}
Background: The optimal anchor density in adolescent idiopathic scoliosis (AIS) surgery to achieve good curve correction remains unclear. The purpose of the study is to analyze the correlations between three-dimensional curve correction and anchor density in the pedicle screw-based posterior fusion of AIS.
\end{abstract}

Methods: One hundred and twenty-seven AIS patients receiving primary posterior fusion with pedicle screw instrumentation were retrospectively reviewed. Anchor density (AD) was defined as the screws number per fused spinal segment. The correlations between three-dimensional curve correction radiographic parameters and anchor density were analyzed with subgroup analysis based on different curve types, curve magnitudes, and curve flexibilities. The differences of curve correction parameters between the low-density (AD $\leq 1.4$ ), middle-density $(1.4<A D \leq 1.7)$ and high-density $(A D>1.7)$ groups were also calculated. Independent t-test, analysis of variance (ANOVA), and Pearson's correlation coefficient were used for statistical analysis.

Results: There were no correlations between the anchor density and the coronal curve correction or apical vertebral rotation (AVR) correction. In the sagittal plane, mild positive correlations existed between anchor density and thoracic kyphosis correction in all patients $(r=0.27, p=0.002)$. Subgroup analysis revealed similar mild positive correlations in Lenke $1(r=0.31, p=0.02)$, Lenke $1-3(r=0.27, p=0.01)$, small curves $\left(40^{\circ}-60^{\circ}, r=0.38, p<0.001\right)$, and flexible curves (flexibility $>40 \%, r=0.34, p=0.01$ ).

There were no differences between low-density (mean 1.31), middle-density (mean 1.55), and high-density (mean 1.83) in terms of coronal or axial curve correction parameters. Low-density group has longer fused level (mean difference 2.14, $p=0.001$ ) and smaller thoracic kyphosis correction (mean difference 9.25,$p=0.004$ ) than highdensity group.

Conclusion: In our study, the anchor density was not related to coronal or axial curve corrections. Mild positive correlations with anchor density were found in thoracic kyphosis correction, especially in patients with smaller and flexible curves. Low anchor density with longer fusion level achieves similar curve corrections with middle or high anchor density in adolescent idiopathic scoliosis surgery.

Keywords: Adolescent idiopathic scoliosis, Three-dimensional curve correction, Anchor density, Posterior fusion, Pedicle screw instrumentation, Thoracic kyphosis

\footnotetext{
* Correspondence: polianglai@gmail.com

'Department of Orthopedic Surgery, Chang Gung Memorial Hospital, No. 5,

Fuxing St., Guishan Dist.., 33305 Taoyuan, Taiwan

${ }^{2}$ Bone and Joint Research Center, Chang Gung Memorial Hospital, Taoyuan,

Taiwan

Full list of author information is available at the end of the article
}

(c) The Author(s). 2019 Open Access This article is distributed under the terms of the Creative Commons Attribution 4.0 International License (http://creativecommons.org/licenses/by/4.0/), which permits unrestricted use, distribution, and reproduction in any medium, provided you give appropriate credit to the original author(s) and the source, provide a link to the Creative Commons license, and indicate if changes were made. The Creative Commons Public Domain Dedication waiver (http://creativecommons.org/publicdomain/zero/1.0/) applies to the data made available in this article, unless otherwise stated. 


\section{Background}

Pedicle screw-based instrumentation has gained popularity in surgery of adolescent idiopathic scoliosis (AIS) patients in the past twenty years [1-3]. From a mechanical standpoint, it provides three columns fixation of the vertebrae and shows a better curve correction for AIS patients. However, increased anchor density may lead to higher implant costs, risks of implant malposition, and blood loss [4-6].

AIS curves are complex three-dimensional deformities that require adequate correction in all three dimensions, including coronal, sagittal and axial planes. Controversies still exist in determining the relation between anchor density and curve correction in all three dimensions [7-17]. In a recent consensus, most experienced panelists agreed that less than 1.60 of the anchor density was optimal in treating AIS with smaller major curve $\left(40^{\circ}-70^{\circ}\right)$ [18]. The lower safe limit of anchor density to maintain long-term adequate correction remains to be determined.

The main purpose of the present study is to determine the relations between anchor density and curve correction in coronal, sagittal and axial planes of adolescent idiopathic scoliosis surgery.

\section{Material and methods \\ Patients}

The present retrospective study analyzed a consecutive series of patients with AIS who received posterior fusion and instrumentation at our institute. This study was approved by the Institute Review Board (IRB No. 201701561B0) at our hospital. All of the patients underwent surgery between January 2009 and December 2013. The AIS patients included in this study were 1) aged from 10 to 24 years old, 2) received primary posterior fusion and instrumentation, 3) used all pedicle screw constructs, and 4) followed up for at least 48 months. The patients were excluded if any of the following criteria were met: 1) neuromuscular scoliosis, 2) infantile or juvenile idiopathic scoliosis, 3) received pedicle screws and hooks hybrid fixation, 4) received anterior fusion and instrumentation surgeries, and 5) inadequate follow-up duration.

All of the surgeries were performed by the same surgical team, comprising two attending surgeons and one rotating resident. All scoliotic curves were classified and operated according to the guidelines described by Lenke [19].

\section{Surgical techniques}

The surgical procedures followed the technique described by Suk [20]. A standard midline approach was applied with the patient in the prone position. Intraoperative posteroanterior and lateral radiographs were used to assess the positions of $\mathrm{K}$-wires as pedicle screw entry points. The pedicle screws were then inserted with the guidance of K-wires. The implant distribution was preoperatively determined based on curve magnitude, flexibility and fusion length. The preoperative planned upper instrumented vertebra (UIV), lower instrumented vertebra (LIV) would be fully inserted with pedicle screws at the bilateral pedicles. The preoperative determined reduction side (concave or convex) pedicles were inserted with pedicle screws every level or every other level. The contralateral side pedicles were inserted with pedicle screws every two or three levels. Each fused vertebra had at least one pedicle screw. After confirmation of pedicle screws positions with portable X-ray device, rods were applied on the tulips of the screws. Partial facetectomy was performed at every level for local bone grafting. The major curve deformities were corrected by the derotation of the titanium rods, with hyperkyphotic contour rods were used at the concave side. Compression and distraction methods were used to assist correction of the proximal thoracic deformities and achieve shoulder balance. For deformities larger than $90^{\circ}$, cantilever bending was utilized for achieving better correction, especially in Lenke type 4 curves. The fixation constructs were all pedicle screw-based constructs. Crosslinks were routinely used at proximal and distal ends to reinforce the structure of the fixation construct. Posterior fusion was performed using local autologous bone chips and supplementary bone substitutes. The wounds were then closed in layers with the placement of a drainage tube.

Electroneurophysiological monitoring was performed during the operation. Standing and walking were encouraged on the second postoperative day with the application of thoracolumbar orthosis.

\section{Assessment of the results}

The medical records of all patients were collected under the approval of the Institutional Review Board of our hospital. The curve patterns were classified according to the Lenke classification [19]. Preoperative and postoperative 2-year whole spinal column standing anteroposterior and lateral images were reviewed and analyzed to compare multiple radiographic parameters. For the coronal plane, major curve and structural curve Cobb angles were measured and recorded. Corresponding curve flexibilities were analyzed based on preoperative supine bending views of both sides. The curve correction rate and curve flexibility were defined as follows:

$$
\begin{aligned}
& \text { Correction rate }(\%)=\frac{\text { Preoperative Cobb angle }- \text { Postoperative Cobb angle }}{\text { Preoperative Cobb angle }} \times 100 \% \\
& \text { Flexibility }(\%)=\frac{\text { Preoperative Cobb angle }- \text { Side bending Cobb angle }}{\text { Preoperative Cobb angle }} \times 100 \%
\end{aligned}
$$

To account for differences in curve flexibility, the Cincinnati curve correction index (CI) was analyzed, as described by Vora et al. [21]: 


$$
\begin{gathered}
\text { Cincinnati Correction index }(\mathrm{CI}) \\
=\frac{\text { Curve correction rate }(\%)}{\text { Curve flexibility }(\%)}
\end{gathered}
$$

Thoracic kyphosis (TK= T5-T12 Cobb angle) and lumbosacral lordosis (LL = L1-S1 Cobb angle) were measured in the sagittal plane. The axial rotation of major curve apical vertebra was measured using the Nash-Moe method [22]. The positions of major curve apical vertebral pedicles on standing whole spine anteroposterior radiographs were classified into the following 5 grades: 0 , no asymmetry of the positions of pedicles; 1 , the convex pedicle has medial migration and concave pedicle starts to disappear; 2 , the convex pedicle continues medially migrating, and the concave pedicle has disappeared; 3 , the convex pedicle touches the midline of the vertebral body; and 4, the convex pedicle migrates past the midline of the vertebral body. Coronal balance, sagittal balance, and apical vertebral translation were also calculated. Anchor density (AD) was defined as the number of total inserted pedicle screws divided by fusion levels.

\section{Statistical analysis}

The correlation between the anchor density and the postoperative 2-year curve correction parameters in three dimensions (major curve correction rate, correction index, thoracic kyphosis correction, lumbar lordosis correction, apical vertebral rotation correction, and apical vertebral translation correction) were calculated using Pearson's correlation coefficient. Subgroup analyses based on different curve types, curve magnitudes, and curve flexibilities were performed as followed: [1] All patients [2]; Main thoracic curves (MT, Lenke 1) [3]; Major MT curves \pm other minor structural curves (Lenke 1-3) [4]; Thoracolumbar/lumbar curves (TL/L, Lenke 5) [5]; Major TL/L curves \pm other minor structural curves (Lenke 5,6) [6]; Double structural MT and TL/L curves (Lenke 3,4,6) [7]; Small curve (major curve between $40^{\circ}$ to $60^{\circ}$ ) [8]; Large curve (major curve over $60^{\circ}$ ) [9]; Stiff curve (flexibility $\leq 40 \%$ ) [10]; Flexible curve (flexibility $>40 \%$ ).

To further investigate the impact of anchor density on curve correction, patients were divided into lowdensity ( $\mathrm{AD} \leq 1.4)$, middle-density $(1.4<\mathrm{AD} \leq 1.7)$ and high-density $(A D>1.7)$ groups (Fig. 1a, 1b \& 1c) in different patient subgroups: [1] All patients [2]; Lenke 1-3 [3]; Lenke 1 patients. The statistical differences between low-density, middle-density, and high-density groups were tested using one-way analysis of variance (ANOVA) with Scheffé post-hoc tests. A $p$ value of less than 0.05 was considered statistically significant in this study. All statistical tests were performed using SPSS v25 (IBM-SPSS, Armonk, NY).

\section{Results}

A total of 127 patients (17 males and 110 females) aged 14.4 years old at the time of surgery were enrolled in the present study. The numbers of Lenke type 1, 2, 3, 4, 5 and 6 curve patients were 59,19,12, 6, 22 and 9, respectively. The mean anchor density of all patients was 1.60 (ranging from 1.14 to 2.0). The demographic data and radiographic parameters for all patients and Lenke 1-6 subgroup patients are shown in Table 1.

\section{Correlations between anchor density and curve correction in different curve types}

According to the results shown in Table 2, anchor density was not correlated with coronal curve correction or apical vertebral rotation (AVR) correction (correction rate: $\mathrm{r}=-0.01, p=0.88$; correction index: $\mathrm{r}=-0.04, p=$ 0.63 ; AVR correction: $\mathrm{r}=0.03, p=0.75$ ) in all patients. Nevertheless, no correlations existed between anchor density and the two-plane corrections in all of the subgroup analyses.

As for the sagittal curve correction, mild but positive correlations existed between anchor density and thoracic kyphosis correction in all patients $(\mathrm{r}=0.27, p=0.002)$, Lenke 1 patients $(\mathrm{r}=0.31, p=0.02)$ and patients with major MT curves \pm other minor structural curves (Lenke $1-3 ; \mathrm{r}=0.27, p=0.01$ ). However, there were no correlations between anchor density and lumbar lordosis correction in Lenke $5(\mathrm{r}=0.16, p=0.47)$ and patients with major TL/L curves \pm other minor structural curves (Lenke5-6; $\mathrm{r}=0.06, p=0.74$ ).

In Lenke 5 patients, apical vertebral translation was positively correlated with anchor density $(\mathrm{r}=0.43, p=$ $0.03)$. No correlations between apical vertebral translation and anchor density were found in the remaining subgroups.

For patients with double structural MT and TL/L curves (Lenke 3,4 \& 6), there were no correlations between anchor density and curve corrections in all dimensions. (Table 2).

\section{Correlations between anchor density and curve correction in different curve magnitudes and flexibilities}

No correlations existed between anchor density and coronal curve correction, correction index, lumbar lordosis (LL) correction, apical vertebral rotation (AVR) correction, or apical vertebral translation (AVT) correction in subgroup analysis of different curve magnitudes (small curves between $40^{\circ}-60^{\circ}$, and large curves $>60^{\circ}$ ) and flexibilities (stiff curves with flexibility $\leq 40 \%$, and flexible curves with flexibility $>40 \%$ ). (Table 3 ).

Thoracic kyphosis (TK) correction was correlated with anchor density in small curves $(\mathrm{r}=0.38, p<0.001)$ and flexible curves $(\mathrm{r}=0.34, p=0.01)$. No correlations were observed between thoracic kyphosis (TK) correction and 

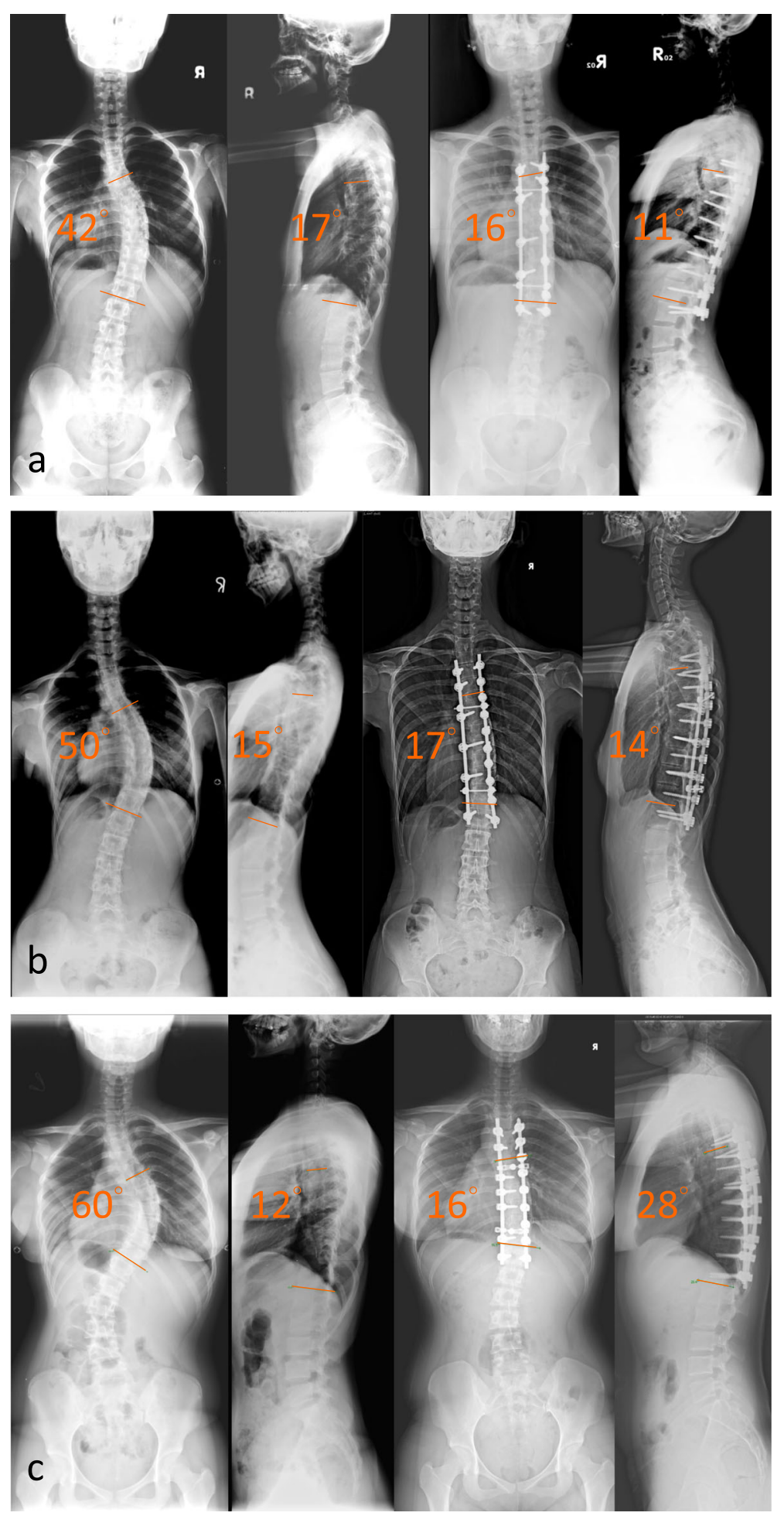

Fig. 1 Low-density, Middle-density and High-density screw constructs. a: A patient with Lenke 1 AIS received posterior fusion of T4-L1 with a low-density construct (Anchor density $=1.20$ ) of pedicle screw instrumentation. b: A patient with Lenke 1 AIS received posterior fusion of T4-L1 with a middle-density construct (Anchor density $=1.60$ ) of pedicle screw instrumentation. c: A patient with Lenke 1 AlS received posterior fusion of T4-T12 with a high-density construct (Anchor density $=1.78$ ) of pedicle screw instrumentation 


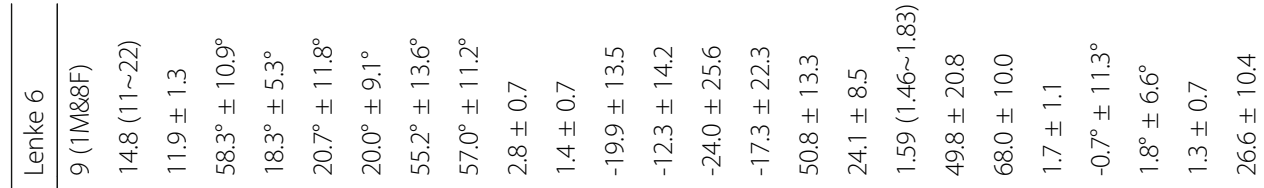

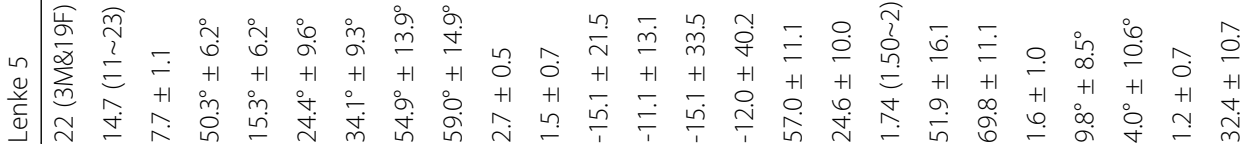

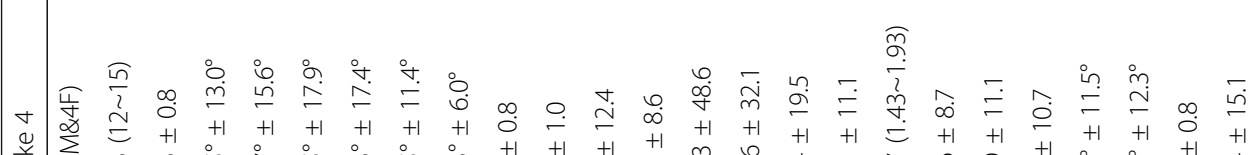

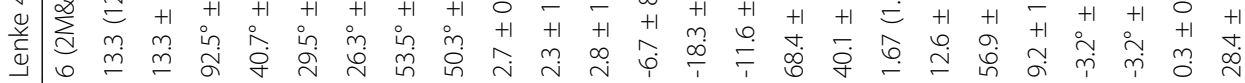

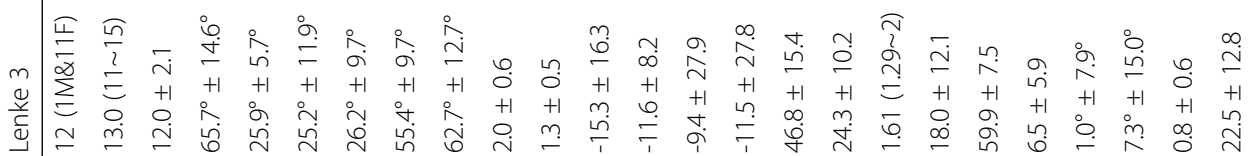

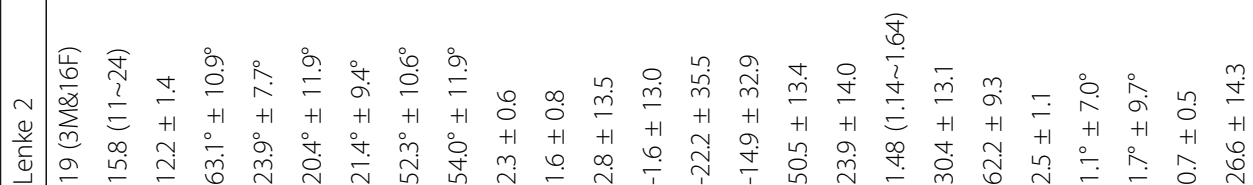

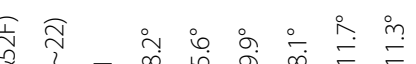

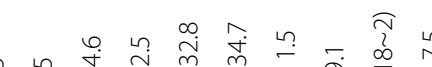

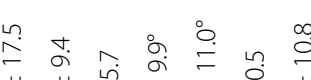

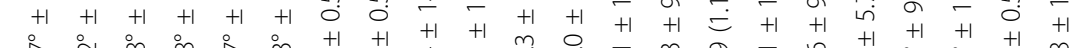

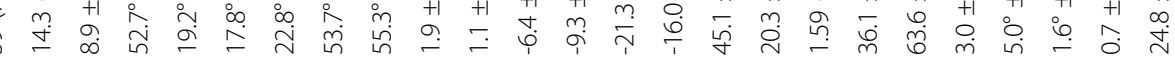

(1)

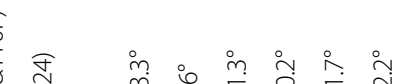

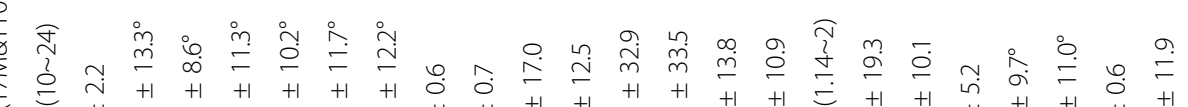

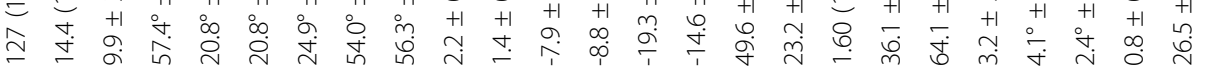

.

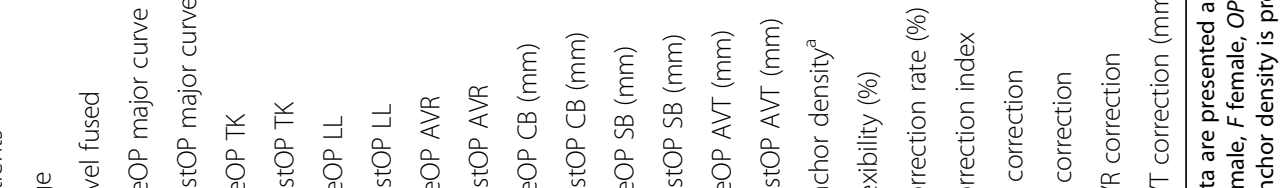

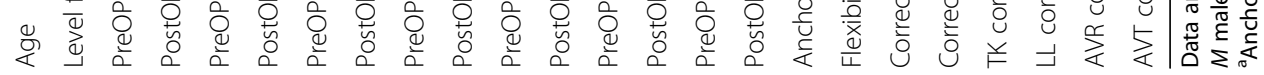


Table 2 Correlation coefficient between anchor density and all correction parameters

\begin{tabular}{|c|c|c|c|c|c|c|c|c|c|c|c|c|}
\hline \multirow[t]{2}{*}{ Parameters } & \multicolumn{2}{|c|}{ All $(\mathrm{N}=127)$} & \multicolumn{2}{|c|}{$\begin{array}{l}\text { Lenke } 1(\mathrm{~N}= \\
\text { 59) }\end{array}$} & \multicolumn{2}{|c|}{$\begin{array}{l}\text { Major MT (Lenke 1-3)(N= } \\
\text { 90) }\end{array}$} & \multicolumn{2}{|c|}{$\begin{array}{l}\text { Lenke } 5(\mathrm{~N}= \\
\text { 22) }\end{array}$} & \multicolumn{2}{|c|}{$\begin{array}{l}\text { Major TL/L (Lenke 5,6)( } \mathrm{N}= \\
\text { 31) }\end{array}$} & \multicolumn{2}{|c|}{$\begin{array}{l}\text { Double T\&L (Lenke 3,4,6) }(\mathrm{N}= \\
\text { 27) }\end{array}$} \\
\hline & $r$ & $p$ & $r$ & $p$ & $r$ & $p$ & $r$ & $p$ & $r$ & $p$ & $r$ & $p$ \\
\hline Correction rate & -0.01 & 0.88 & -0.04 & 0.76 & -0.06 & 0.59 & -0.35 & 0.11 & -0.19 & 0.32 & -0.30 & 0.12 \\
\hline Correction index & -0.04 & 0.63 & -0.04 & 0.75 & -0.01 & 0.97 & -0.26 & 0.24 & -0.07 & 0.71 & -0.02 & 0.92 \\
\hline TK correction & 0.27 & 0.002 & 0.31 & 0.02 & 0.27 & 0.01 & 0.40 & 0.06 & 0.33 & 0.07 & 0.12 & 0.56 \\
\hline LL correction & -0.07 & 0.45 & -0.08 & 0.56 & -0.11 & 0.32 & 0.16 & 0.47 & 0.06 & 0.74 & -0.22 & 0.27 \\
\hline AVR correction & 0.03 & 0.75 & -0.24 & 0.07 & -0.14 & 0.18 & 0.16 & 0.48 & 0.09 & 0.62 & -0.14 & 0.47 \\
\hline AVT correction & 0.01 & 0.90 & -0.12 & 0.37 & -0.15 & 0.15 & 0.43 & 0.03 & 0.31 & 0.10 & -0.02 & 0.90 \\
\hline
\end{tabular}

Major MT major main thoracic, Major TL/L major thoracolumbar/lumbar, Double T\&L double thoracic and lumbar; $r$ Pearson's correlation coefficient; $p p$ value $T K$ thoracic kyphosis, LL lumbar lordosis, AVR apical vertebral rotation, AVT apical vertebral translation

anchor density in large curves $(\mathrm{r}=-0.06, p=0.72)$ and stiff curves $(\mathrm{r}=0.24, p=0.06)$.

\section{Comparisons between different anchor density subgroups} The comparisons of fused level, flexibility and curve correction parameters between low-density, middle-density and high-density screw constructs are presented in Table 4. There were no differences between low-density, middledensity, and high-density in terms of coronal or axial curve correction parameters in all patients, Lenke 1-3 patients, and Lenke 1 patients. Significant differences between the three groups were observed in fused level and thoracic kyphosis correction.

Post-hoc Scheffé tests between different anchor density groups were shown in Table 5 . In all patients, high-density group has significantly shorter fused level than low-density (mean difference 2.14, $p=0.001$ ) and middle-density (mean difference 2.09, $p<0.001$ ) groups, and larger thoracic kyphosis correction than low-density group (mean difference 9.25,$p=0.004$ ). Similar differences were observed in Lenke 1-3 patients, although low-density group has less thoracic kyphosis correction (mean difference $6.03^{\circ}, p=0.03$ ) despite comparable fused level (mean difference 0.17, $p=0.94$ ). Whereas in Lenke 1 patients, low-density has less thoracic kyphosis correction compared to middle- density (mean difference $10.21^{\circ}, p=0.01$ ) and highdensity (mean difference $10.60^{\circ}, p=0.01$ ) groups without significant fused level differences.

\section{Discussion}

Pedicle screws instrumentation and posterior fusion have become the treatment of choice in AIS surgery [1-3]. Although, with the advancement of pedicle screw insertion techniques and electroneurophysiological monitoring systems, neurologic complications, increased intraoperative blood loss and implant costs remained a concern for spinal surgeons [4-6]. Correcting scoliotic deformity in the coronal plane is one of the earliest established surgical goals for AIS patients. The correlation of anchor density and AIS coronal curve correction has been widely studied and reported over the past decades. However, studies have shown contradictory results regarding the correlation of anchor density and coronal curve correction in AIS surgery [7-17].

Several studies have shown positive correlations between AIS coronal curve correction and anchor density. Clements et al. [7] reviewed 250 major thoracic and 42 major lumbar curves within all 6 Lenke types and observed weak but significant correlations with mixed types of implants. Yang et al. [9] proposed a similar weak correlation within 58 Lenke $1 \mathrm{~A}$ and $1 \mathrm{~B}$ patients. Chen

Table 3 Correlations between anchor density and curve correction in different curve sizes and flexibilities

\begin{tabular}{|c|c|c|c|c|c|c|c|c|}
\hline \multirow[t]{2}{*}{ Parameters } & \multicolumn{2}{|c|}{$\begin{array}{l}\text { Small curve } \\
\left(40^{\circ}-60^{\circ}\right)(\mathrm{N}=89)\end{array}$} & \multicolumn{2}{|c|}{$\begin{array}{l}\text { Large curve } \\
\left(>60^{\circ}\right)(\mathrm{N}=38)\end{array}$} & \multicolumn{2}{|c|}{$\begin{array}{l}\text { Stiff curve (flexibility } \leq 40 \%) \\
(\mathrm{N}=78)\end{array}$} & \multicolumn{2}{|c|}{$\begin{array}{l}\text { Flexible curve } \\
\text { (flexibility }>40 \%)(\mathrm{N}=49)\end{array}$} \\
\hline & $r$ & $p$ & $r$ & $p$ & $r$ & $p$ & $r$ & $p$ \\
\hline Correction rate & -0.04 & 0.69 & -0.10 & 0.54 & -0.04 & 0.73 & -0.05 & 0.71 \\
\hline Correction index & -0.03 & 0.78 & 0.02 & 0.92 & -0.04 & 0.71 & -0.15 & 0.30 \\
\hline TK correction & 0.38 & $<0.001$ & -0.06 & 0.72 & 0.24 & 0.06 & 0.34 & 0.01 \\
\hline LL correction & 0.01 & 0.90 & -0.15 & 0.39 & -0.07 & 0.54 & 0.06 & 0.69 \\
\hline AVR correction & -0.04 & 0.70 & -0.01 & 0.95 & -0.08 & 0.48 & 0.07 & 0.66 \\
\hline AVT correction & 0.05 & 0.65 & 0.01 & 0.96 & -0.12 & 0.29 & 0.23 & 0.12 \\
\hline
\end{tabular}

$r$ Pearson's correlation coefficient, $p p$ value

$T K$ thoracic kyphosis, LL lumbar lordosis, AVR apical vertebral rotation, AVT apical vertebral translation 
Table 4 Different anchor density groups comparisons

\begin{tabular}{|c|c|c|c|c|}
\hline & $\begin{array}{l}\text { Low } \\
\mathrm{AD} \leq 1.4\end{array}$ & $\begin{array}{l}\text { Middle } \\
1.4<\mathrm{AD} \leq 1.7\end{array}$ & $\begin{array}{l}\text { High } \\
A D>1.7\end{array}$ & $p$ value \\
\hline \multicolumn{5}{|l|}{ All patients } \\
\hline Patients & 16 (2M\&14F) & 73 (7M\&66F) & 38 (8M\&30F) & \\
\hline Anchor density & $1.31 \pm 0.07$ & $1.55 \pm 0.08$ & $1.83 \pm 0.10$ & $<0.001$ \\
\hline Fused level & $10.6 \pm 2.1$ & $10.5 \pm 2.1$ & $8.4 \pm 1.7$ & $<0.001$ \\
\hline Flexibility (\%) & $32.0 \pm 12.6$ & $35.5 \pm 20.8$ & $38.9 \pm 18.4$ & 0.45 \\
\hline Correction rate (\%) & $64.3 \pm 9.8$ & $64.1 \pm 9.5$ & $63.9 \pm 11.3$ & 0.99 \\
\hline Correction index & $2.5 \pm 1.8$ & $3.8 \pm 6.5$ & $2.4 \pm 2.4$ & 0.36 \\
\hline TK correction & $-1.9^{\circ} \pm 8.8^{\circ}$ & $3.9^{\circ} \pm 9.7^{\circ}$ & $7.3^{\circ} \pm 9.1^{\circ}$ & 0.005 \\
\hline LL correction & $2.4^{\circ} \pm 13.3^{\circ}$ & $2.5^{\circ} \pm 9.8^{\circ}$ & $2.1^{\circ} \pm 12.3^{\circ}$ & 0.99 \\
\hline AVR correction & $0.7 \pm 0.6$ & $0.9 \pm 0.6$ & $0.8 \pm 0.7$ & 0.60 \\
\hline AVT correction (mm) & $29.2 \pm 13.2$ & $25.7 \pm 11.4$ & $26.5 \pm 11.9$ & 0.57 \\
\hline \multicolumn{5}{|l|}{ Lenke 1-3 } \\
\hline Patients & 16 (2M\&14F) & 54 (5M\&49F) & $20(4 \mathrm{M} \& 16 \mathrm{~F})$ & \\
\hline Anchor density & $1.31 \pm 0.07$ & $1.54 \pm 0.07$ & $1.84 \pm 0.10$ & $<0.001$ \\
\hline Fused level & $10.6 \pm 2.1$ & $10.4 \pm 2.0$ & $8.5 \pm 0.9$ & $<0.001$ \\
\hline Flexibility (\%) & $32.0 \pm 12.6$ & $33.2 \pm 18.7$ & $30.9 \pm 16.0$ & 0.88 \\
\hline Correction rate (\%) & $64.3 \pm 9.8$ & $62.4 \pm 9.1$ & $62.8 \pm 9.1$ & 0.77 \\
\hline Correction index & $2.5 \pm 1.8$ & $3.7 \pm 6.5$ & $3.1 \pm 3.1$ & 0.73 \\
\hline TK correction & $-1.9^{\circ} \pm 8.8^{\circ}$ & $4.1^{\circ} \pm 9.6^{\circ}$ & $6.9^{\circ} \pm 6.5^{\circ}$ & 0.01 \\
\hline LL correction & $2.4^{\circ} \pm 13.3^{\circ}$ & $3.5^{\circ} \pm 10.6^{\circ}$ & $-0.5^{\circ} \pm 11.9^{\circ}$ & 0.42 \\
\hline AVR correction & $0.7 \pm 0.6$ & $0.8 \pm 0.5$ & $0.6 \pm 0.5$ & 0.19 \\
\hline AVT correction (mm) & $29.2 \pm 13.2$ & $24.7 \pm 11.2$ & $21.9 \pm 11.6$ & 0.18 \\
\hline \multicolumn{5}{|l|}{ Lenke 1} \\
\hline Patients & 10 (1M\&9F) & 31 (3M\&28F) & 18 (3M\&15F) & \\
\hline Anchor density & $1.33 \pm 0.06$ & $1.53 \pm 0.07$ & $1.82 \pm 0.09$ & $<0.001$ \\
\hline Fused level & $9.2 \pm 0.9$ & $9.1 \pm 1.2$ & $8.3 \pm 0.8$ & 0.03 \\
\hline Flexibility (\%) & $33.3 \pm 13.6$ & $39.1 \pm 19.4$ & $32.3 \pm 15.5$ & 0.37 \\
\hline Correction rate (\%) & $66.5 \pm 9.2$ & $62.6 \pm 10.2$ & $63.7 \pm 8.1$ & 0.53 \\
\hline Correction index & $2.5 \pm 1.8$ & $3.4 \pm 7.8$ & $2.5 \pm 1.4$ & 0.85 \\
\hline TK correction & $-3.6^{\circ} \pm 9.1^{\circ}$ & $6.6^{\circ} \pm 10.5^{\circ}$ & $7.0^{\circ} \pm 6.4^{\circ}$ & 0.008 \\
\hline LL correction & $-2.3^{\circ} \pm 9.7^{\circ}$ & $4.8^{\circ} \pm 10.2^{\circ}$ & $-1.7^{\circ} \pm 11.9^{\circ}$ & 0.06 \\
\hline AVR correction & $0.8 \pm 0.6$ & $0.8 \pm 0.5$ & $0.6 \pm 0.5$ & 0.24 \\
\hline AVT correction (mm) & $27.8 \pm 12.0$ & $25.0 \pm 10.4$ & $22.9 \pm 109$ & 0.52 \\
\hline
\end{tabular}

Data are presented as mean \pm standard deviation (SD)

$A D$ anchor density, $T K$ thoracic kyphosis, LL lumbar lordosis, AVR apical vertebral rotation, AVT apical vertebral translation

et al. [11] further demonstrated a mild correlation $(\mathrm{r}=$ 0.43, $P<0.05$ ) between thoracolumbar/lumbar curve correction and anchor density in 39 Lenke 5 AIS patients. A large series study that consisted of 584 Lenke 1, 245 Lenke 2 and 123 Lenke 5 AIS patients was conducted by Larson et al. [13] The results showed that the high anchor density $(\mathrm{AD} \geq 1.54)$ group has a significantly better coronal curve correction than the low anchor density $(\mathrm{AD}<1.54)$ group in Lenke 1 and Lenke 2 patients, and the significant difference continued to exist within postoperative 2 years. However, Lenke 5 patients did not demonstrate a significant difference in coronal curve correction between high- and low-density groups in the same study. Two studies in 2016 showed support for a positive correlation between coronal curve correction and anchor density. Ketenci et al. [15] studied 76 matched Lenke 1 patients and equally divided these patients into the consecutive pedicle screw group (mean anchor density 2) and the interval pedicle screw group (mean anchor density 1.14). A significantly better coronal 
Table 5 Post-hoc Scheffé tests between different anchor density groups

\begin{tabular}{|c|c|c|c|}
\hline & Groups comparison & Mean difference & $p$ value \\
\hline \multicolumn{4}{|l|}{ All patients } \\
\hline \multirow[t]{3}{*}{ Fused level } & Low > Middle & 0.06 & 0.99 \\
\hline & Low $>$ High & 2.14 & 0.001 \\
\hline & Middle > High & 2.09 & $<0.001$ \\
\hline \multirow[t]{3}{*}{ TK correction } & Low $<$ Middle & $5.64^{\circ}$ & 0.10 \\
\hline & Low $<$ High & $9.25^{\circ}$ & 0.004 \\
\hline & Middle $<$ High & $3.62^{\circ}$ & 0.14 \\
\hline \multicolumn{4}{|l|}{ Lenke 1-3 } \\
\hline \multirow[t]{3}{*}{ Fused level } & Low > Middle & 0.17 & 0.94 \\
\hline & Low $>$ High & 2.11 & 0.003 \\
\hline & Middle > High & 1.94 & $<0.001$ \\
\hline \multirow[t]{3}{*}{ TK correction } & Low $<$ Middle & $6.03^{\circ}$ & 0.049 \\
\hline & Low $<$ High & $8.79^{\circ}$ & 0.01 \\
\hline & Middle $<$ High & $2.76^{\circ}$ & 0.50 \\
\hline \multicolumn{4}{|l|}{ Lenke 1} \\
\hline \multirow[t]{3}{*}{ Fused level } & Low > Middle & 0.14 & 0.94 \\
\hline & Low > High & 0.87 & 0.10 \\
\hline & Middle > High & 0.73 & 0.06 \\
\hline \multirow[t]{3}{*}{ TK correction } & Low $<$ Middle & $10.21^{\circ}$ & 0.01 \\
\hline & Low $<$ High & $10.60^{\circ}$ & 0.01 \\
\hline & Middle $<$ High & $0.39^{\circ}$ & 0.99 \\
\hline
\end{tabular}

Note: only the parameters with significant difference in analysis of variance were listed

TK thoracic kyphosis; Low: anchor density $\leq 1.4$; Middle: $1.4<$ anchor density $\leq$ 1.7; High: anchor density $>1.7$

and rotational correction of thoracic curve was observed in the consecutive pedicle screw group. Nevertheless, Mac-Thiong et al. [17] reviewed the coronal main curve and main thoracic curve correction of 137 AIS patients. The results showed that the curve correction of anchor density $<1.4$ was significantly inferior to the curve correction of anchor density $\geq 1.8$, whereas the curve correction of anchor density between 1.4 to 1.8 showed comparable results to an anchor density $\geq 1.8$.

In contrast, several studies reported no correlations between coronal curve correction and anchor density. Quan et al. [8] reviewed 49 Lenke 1 patients and found no correlation between anchor density and coronal curve correction. Bharucha et al. [10] divided 91 Lenke 1 patients into 34 high-density and 57 low-density patients based on mean anchor density (1.3), and no differences of curve correction, thoracic kyphosis or apical thoracic rotation were observed. Gebhard et al. [12] also found no correlation between main thoracic curve correction and anchor density within 119 AIS patients. Rushton et al. [14] demonstrated no correlation between correction of coronal curve, thoracic kyphosis or lumbar lordosis in 106 AIS patients (78 Lenke 1). Kempaninem et al. [16] compared 26 high-density (mean anchor density 1.68) AIS patients with 26 low-density (mean anchor density 1.28) AIS patients and found no differences in major MT curve correction, coronal balance, sagittal balance or apical vertebral translation.

Our study results were more compatible with studies reporting no correlation between anchor density and coronal curve correction $[8,10,12,14,16]$. The coronal curve correction parameters in our study were not correlated with anchor density in all patients subgroups, with the account of different curve types, curve magnitudes and curve flexibilities. (Tables 2 \& 3) Similarly, there were no differences between low-density, middledensity and high-density groups for coronal curves correction. (Table 4).

Spinal surgeons were more aware of the correction of thoracic kyphosis and lumbar lordosis since the introduction of three-dimensional correction concept of AIS surgery [1, 23]. Furthermore, hypokyphosis has been associated with pulmonary function compromise and risk of future proximal junctional kyphosis in AIS patients [24, 25]. Maintaining adequate lumbar lordosis is also essential to balanced sagittal profiles in longsegments instrumented spinal surgery [26]. Larson et al. [13] found high anchor density was associated with postoperative hypokyphosis in Lenke 1,2 AIS patients, while several other studies reported no correlations between anchor density and thoracic kyphosis/lumbar lordosis correction $[14,15,17]$.

In our study, mild positive correlation existed between anchor density and thoracic kyphosis correction in all patients, Lenke 1 patients, and Lenke 1-3 patients. (Table 2) When divided into low-density, middle-density, and highdensity groups, the differences in thoracic kyphosis correction was accompanied by fused level differences. (Table 4) Post-hoc Scheffé tests revealed that with about two addition fusion levels, the thoracic kyphosis correction was about $9^{\circ}$ less in low-density group, as compared to high-density group. (Table 5) However, the thoracic kyphosis corrections were about $10^{\circ}$ less with no significant differences in fusion level in low-density group when comparing to other two groups in Lenke 1 patients.

Several factors influence thoracic kyphosis corrections included preoperative thoracic kyphosis, main thoracic curve flexibility, anchor density of concave side, rod materials and facetectomy levels [27-29]. Although statistical radiographic differences of thoracic kyphosis correction were observed between low-density and other two groups, clinical significances of thoracic kyphosis correction were not reached since the mean differences were only $5^{\circ}-10^{\circ}$ between the three groups.

Studies have shown no significant correlations between anchor density and lumbar lordosis in instrumented 
thoracolumbar/lumbar curves $[11,13]$, which is compatible to our study in both Lenke 5 and patients with major TL/L curves \pm other minor structural curves (Lenke 5-6). (Table 2) For patients with instrumentations in both structural thoracic and structural thoracolumbar/lumbar curves (Lenke 3,4,6), anchor density was not correlated with thoracic kyphosis $(r=-0.12, p=0.55)$ and lumbar lordosis $(\mathrm{r}=-0.22, p=0.27)$ based on our study statistics.

The correction of the axial rotation of the deformed vertebrae is one of the key components of AIS surgery. Few studies have examined the relationship between anchor density and the rotational correction of axial plane. Bharucha et al. [10] reported no differences of trunk rotation correction between anchor density above or below 1.3 (mean anchor density of all 91 patients). Whereas Ketenci et al. [15] found consecutive pedicle screws construct (anchor density 2.0) has significant better apical vertebral rotation correction over interval pedicle screw construct (anchor density 1.14). In our study, there was no correlation between anchor density and apical vertebral rotation in all patients and all subgroups of different curve types, curve magnitudes and curve flexibilities. (Tables $2 \& 3$ ) Nevertheless, no differences were observed between low-density, middle-density, and high-density groups in terms of apical vertebral rotation correction $(p=0.60)$ in all patients. (Table 4).

To investigate the effect of anchor density in different curve magnitudes and flexibilities, Pearson correlation coefficients between anchor density and curve correction were calculated in small curves $\left(40^{\circ}-60^{\circ}\right)$, large curves (> $60^{\circ}$ ), stiff curves (flexibility $\leq 40 \%$ ) and flexible curves (flexibility $>40 \%$ ) (Table 3). From the literatures [30, 31], the flexibility assessed by supine bending views were averaged more than $40 \%$, which was set as the cutoff value between stiff curves and flexible curves in our study. In large and stiff curves, no correlations between anchor density and all curve correction parameters were observed. While thoracic kyphosis was positively correlated with anchor density in small curves $(r=0.38, p<0.001)$ and flexible curves $(\mathrm{r}=0.34, p=0.01)$. Therefore, for small and flexible curves, increase the anchor density may result in more thoracic kyphosis correction. But the difference may not be obvious when the anchor density exceeds more than 1.7. However, for large and stiff curves, the anchor density was not correlated with all curve correction parameters, other correction techniques or longer fusion length were typically utilized to achieve adequate correction.

There were several limitations of this study, including the retrospective nature and lack of patient-reported outcome evaluations. The patients' standing postures were not standardized in erect whole spine lateral view, which resulted in interpretation and reporting bias. Sagittal radiographic parameters were easily influenced by the patients' positioning and motion [32, 33]. In addition, evaluating the axial vertebral rotation with the Nash-Moe method may not reflect the true axial rotation, since the classification only divides the rotations into four categories [22]. Further prospective studies and long-term surgical outcome comparisons can provide stronger evidence to clarify the true relationships between three-dimensional curve correction and anchor density.

\section{Conclusion}

In our study, the anchor density was not related to coronal or axial curve corrections. Mild positive correlations with anchor density were found in thoracic kyphosis correction, especially in patients with smaller and flexible curves. Low anchor density with longer fusion level achieves similar curve corrections with middle or high anchor density in adolescent idiopathic scoliosis surgery. Therefore, spinal surgeons should consider the influences of anchor density on correcting deformities when planning the distributions of implants preoperatively.

\section{Abbreviations}

AD: Anchor density; AIS: Adolescent idiopathic scoliosis; AVR: Apical vertebral rotation; Cl: Cincinnati curve correction index; LL: Lumbosacral lordosis; MT: Main thoracic; PT: Proximal thoracic; TK: Thoracic kyphosis; TL/ L: Thoracolumbar/Lumbar

\section{Acknowledgments \\ The authors thank Biostatistical Center for Clinical Research, Chang Gung Memorial Hospital at Linkou, for statistical consultation.}

\section{Authors' contributions}

YCY, a resident, contributed to data collection and drafted the manuscript. CCN and LHC, senior surgeons, proposed valuable suggestions and participated in revising the manuscript. WJC, a senior surgeon, performed surgery and clinical examinations at follow-up. PLL, a senior surgeon, performed surgery, initiated the study design and commented on the results. All authors read and approved the final manuscript.

Funding

No funding support.

\section{Availability of data and materials}

The datasets of this study are available from the corresponding author on reasonable request.

Ethics approval and consent to participate

This study was approved by the Institutional Review Board of Chang Gung Memorial Hospital at Linkou, Taoyuan, Taiwan. The need for patient consent was waived by the committee. (IRB No. 201701561B0).

Consent for publication

Not applicable.

\section{Competing interests}

All the authors have no conflicts of interest to disclose.

\section{Author details}

'Department of Orthopedic Surgery, Chang Gung Memorial Hospital, No. 5, Fuxing St., Guishan Dist., 33305 Taoyuan, Taiwan. ${ }^{2}$ Bone and Joint Research Center, Chang Gung Memorial Hospital, Taoyuan, Taiwan. ${ }^{3}$ College of Medicine, Chang Gung University, Taoyuan, Taiwan. ${ }^{4}$ Department of Orthopedic Surgery, Chung Shan Hospital, Taipei, Taiwan. 
Received: 21 March 2019 Accepted: 20 September 2019

Published online: 27 October 2019

\section{References}

1. Kim YJ, Lenke LG, Kim J, Bridwell KH, Cho SK, Cheh G, et al. Comparative analysis of pedicle screw versus hybrid instrumentation in posterior spinal fusion of adolescent idiopathic scoliosis. Spine. 2006;31(3):291-8.

2. Asghar J, Samdani AF, Pahys JM, D'Andrea LP, Guille JT, Clements DH, et al. Computed tomography evaluation of rotation correction in adolescent idiopathic scoliosis: a comparison of an all pedicle screw construct versus a hook-rod system. Spine. 2009;34(8):804-7.

3. Yilmaz G, Borkhuu B, Dhawale AA, Oto M, Littleton AG, Mason DE, et al. Comparative analysis of hook, hybrid, and pedicle screw instrumentation in the posterior treatment of adolescent idiopathic scoliosis. J Pediatr Orthop. 2012;32(5):490-9.

4. Hicks JM, Singla A, Shen FH, Arlet V. Complications of pedicle screw fixation in scoliosis surgery: a systematic review. Spine. 2010;35(11):E465-70.

5. Ledonio CG, Polly DW Jr, Vitale MG, Wang Q, Richards BS. Pediatric pedicle screws: comparative effectiveness and safety: a systematic literature review from the Scoliosis Research Society and the pediatric Orthopaedic Society of North America task force. J Bone Joint Surg Am. 2011;93(13):1227-34.

6. Larson AN, Polly DW Jr, Ackerman SJ, Ledonio CG, Lonner BS, Shah SA, et al. What would be the annual cost savings if fewer screws were used in adolescent idiopathic scoliosis treatment in the US? Journal of neurosurgery Spine. 2015:1-8

7. Clements DH, Betz RR, Newton PO, Rohmiller M, Marks MC, Bastrom T. Correlation of scoliosis curve correction with the number and type of fixation anchors. Spine. 2009;34(20):2147-50.

8. Quan GM, Gibson MJ. Correction of main thoracic adolescent idiopathic scoliosis using pedicle screw instrumentation: does higher implant density improve correction? Spine. 2010;35(5):562-7.

9. Yang S, Jones-Quaidoo SM, Eager M, Griffin JW, Reddi V, Novicoff W, et al. Right adolescent idiopathic thoracic curve (Lenke $1 \mathrm{a}$ and B): does cost of instrumentation and implant density improve radiographic and cosmetic parameters? European spine journal : official publication of the European Spine Society, the European Spinal Deformity Society, and the European Section of the Cervical Spine Research Society. 2011;20(7):1039-47.

10. Bharucha NJ, Lonner BS, Auerbach JD, Kean KE, Trobisch PD. Lowdensity versus high-density thoracic pedicle screw constructs in adolescent idiopathic scoliosis: do more screws lead to a better outcome? The spine journal : official journal of the North American Spine Society. 2013;13(4):375-81.

11. Chen J, Yang C, Ran B, Wang Y, Wang C, Zhu X, et al. Correction of Lenke 5 adolescent idiopathic scoliosis using pedicle screw instrumentation: does implant density influence the correction? Spine. 2013;38(15):E946-51.

12. Gebhart S, Alton TB, Bompadre V, Krengel WF. Do anchor density or pedicle screw density correlate with short-term outcome measures in adolescent idiopathic scoliosis surgery? Spine. 2014:39(2):E104-10.

13. Larson AN, Polly DW Jr, Diamond B, Ledonio C, Richards BS 3rd, Emans $\mathrm{JB}$, et al. Does higher anchor density result in increased curve correction and improved clinical outcomes in adolescent idiopathic scoliosis? Spine. 2014;39(7):571-8.

14. Rushton PR, Elmalky M, Tikoo A, Basu S, Cole AA, Grevitt MP. The effect of metal density in thoracic adolescent idiopathic scoliosis. European spine journal : official publication of the European Spine Society, the European Spinal Deformity Society, and the European Section of the Cervical Spine Research Society. 2015.

15. Ketenci IE, Yanik HS, Demiroz S, Ulusoy A, Erdem S. Three-dimensional correction in patients with Lenke 1 adolescent idiopathic scoliosis: comparison of consecutive versus interval pedicle screw instrumentation. Spine. 2016:41(2):134-8.

16. Kemppainen JW, Morscher MA, Gothard MD, Adamczyk MJ, Ritzman TF. Evaluation of limited screw density pedicle screw constructs in posterior fusions for adolescent idiopathic scoliosis. Spine Deform. 2016;4(1):33-9.

17. Mac-Thiong JM, Ibrahim S, Parent S, Labelle H. Defining the number and type of fixation anchors for optimal main curve correction in posterior surgery for adolescent idiopathic scoliosis. The spine journal : official journal of the North American Spine Society. 2016.

18. de Kleuver M, Lewis SJ, Germscheid NM, Kamper SJ, Alanay A, Berven SH, et al. Optimal surgical care for adolescent idiopathic scoliosis: an international consensus. European spine journal : official publication of the European Spine
Society, the European Spinal Deformity Society, and the European Section of the Cervical Spine Research Society. 2014;23(12):2603-18.

19. Lenke LG. The Lenke classification system of operative adolescent idiopathic scoliosis. Neurosurg Clin N Am. 2007:18(2):199-206.

20. Suk SI, Kim JH, Kim SS, Lim DJ. Pedicle screw instrumentation in adolescent idiopathic scoliosis (AIS). European spine journal : official publication of the European Spine Society, the European Spinal Deformity Society, and the European Section of the Cervical Spine Research Society. 2012;21(1):13-22.

21. Vora V, Crawford A, Babekhir N, Boachie-Adjei O, Lenke L, Peskin M, et al. A pedicle screw construct gives an enhanced posterior correction of adolescent idiopathic scoliosis when compared with other constructs: myth or reality. Spine. 2007;32(17):1869-74.

22. Nash $\mathrm{CL} \mathrm{Jr}$, Moe JH. A study of vertebral rotation. J Bone Joint Surg Am. 1969;51(2):223-9.

23. Mac-Thiong JM, Labelle H, Charlebois M, Huot MP, de Guise JA. Sagittal plane analysis of the spine and pelvis in adolescent idiopathic scoliosis according to the coronal curve type. Spine. 2003:28(13):1404-9.

24. Newton PO, Faro FD, Gollogly S, Betz RR, Lenke LG, Lowe TG. Results of preoperative pulmonary function testing of adolescents with idiopathic scoliosis. A study of six hundred and thirty-one patients. J Bone Joint Surg Am. 2005;87(9):1937-46.

25. Yaszay B, Bastrom TP, Bartley CE, Parent S, Newton PO. The effects of the threedimensional deformity of adolescent idiopathic scoliosis on pulmonary function. European spine journal : official publication of the European Spine Society, the European Spinal Deformity Society, and the European Section of the Cervical Spine Research Society. 2017:26(6):1658-64.

26. Yang X, Liu L, Song Y, Zhou C, Zhou Z, Wang L, et al. Pre- and postoperative spinopelvic sagittal balance in adolescent patients with lenke type 5 idiopathic scoliosis. Spine. 2015;40(2):102-8.

27. Sudo H, Abe Y, Kokabu T, Ito M, Abumi K, Ito Y, et al. Correlation analysis between change in thoracic kyphosis and multilevel facetectomy/ screw density in main thoracic adolescent idiopathic scoliosis surgery. The spine journal : official journal of the North American Spine Society. 2016.

28. Kokabu T, Sudo H, Abe Y, Ito M, Ito YM, Iwasaki N. Effects of multilevel Facetectomy and screw density on postoperative changes in spinal rod contour in thoracic adolescent idiopathic scoliosis surgery. PLoS One. 2016; 11(8):e0161906.

29. Luo M, Jiang H, Wang W, Li N, Shen M, Li P, et al. Influence of screw density on thoracic kyphosis restoration in hypokyphotic adolescent idiopathic scoliosis. BMC Musculoskelet Disord. 2017;18(1):526.

30. Klepps SJ, Lenke LG, Bridwell KH, Bassett GS, Whorton J. Prospective comparison of flexibility radiographs in adolescent idiopathic scoliosis. Spine. 2001;26(5):E74-9.

31. He C, Wong MS. Spinal flexibility assessment on the patients with adolescent idiopathic scoliosis: a literature review. Spine. 2018;43(4):E250-e8.

32. Pan F, Firouzabadi A, Reitmaier S, Zander T, Schmidt H. The shape and mobility of the thoracic spine in asymptomatic adults - a systematic review of in vivo studies. J Biomech. 2018;78:21-35.

33. Hayden AM, Hayes AM, Brechbuhler JL, Israel H, Place HM. The effect of pelvic motion on spinopelvic parameters. The spine journal : official journal of the North American Spine Society. 2018;18(1):173-8.

\section{Publisher's Note}

Springer Nature remains neutral with regard to jurisdictional claims in published maps and institutional affiliations.

\section{Ready to submit your research? Choose BMC and benefit from:}

- fast, convenient online submission

- thorough peer review by experienced researchers in your field

- rapid publication on acceptance

- support for research data, including large and complex data types

- gold Open Access which fosters wider collaboration and increased citations

- maximum visibility for your research: over $100 \mathrm{M}$ website views per year

At $\mathrm{BMC}$, research is always in progress.

Learn more biomedcentral.com/submissions 